\title{
USO DE REDES NEURAIS RECORRENTES NA DETERMINAÇÃO DAS CONSTANTES DE ACIDEZ PARA A 7-EPICLUSIANONA EM MISTURAS ETANOL-ÁGUA
}

\section{Éderson D' Martin Costa e Nelson Henrique Teixeira Lemes*}

Instituto de Ciências Exatas, Universidade Federal de Alfenas, Rua Gabriel Monteiro da Silva, 700, 37130-000 Alfenas - MG, Brasil Marcelo Henrique dos Santos

Instituto de Ciências Farmacêuticas, Universidade Federal de Alfenas, Rua Gabriel Monteiro da Silva, 700, 37130-000 Alfenas - MG, Brasil

\section{João Pedro Braga}

Departamento de Química, Universidade Federal de Minas Gerais, Av. Antônio Carlos, 6627, 31270-901 Belo Horizonte - MG, Brasil

Recebido em 14/2/11; aceito em 15/6/11; publicado na web em 5/8/11

\begin{abstract}
USE OF RECURRENT NEURAL NETWORKS FOR DETERMINATION OF 7-EPICLUSIANONE ACIDITY CONSTANTS IN ETHANOL-WATER MIXTURES. This work propose a recursive neural network to solve inverse equilibrium problem. The acidity constants of 7-epiclusianone in ethanol-water binary mixtures were determined from multiwavelength spectrophotmetric data. A linear relationship between acidity constants and the \%w/v of ethanol in the solvent mixture was observed. The proposed method efficiency is compared with the Simplex method, commonly used in nonlinear optimization techniques. The neural network method is simple, numerically stable and has a broad range of applicability.
\end{abstract}

Keywords: inverse problem; acidity constants; dynamical optimization.

\section{INTRODUÇÃO}

A constante de equilíbrio de um ácido ou base é um parâmetro importante para indicar a extensão de ionização em diferentes condições de $\mathrm{pH}$, com grande importância em muitos processos analíticos, tais como, volumetria de neutralização, cromatografia e extração por solventes. ${ }^{1}$ No meio biológico a constante de equilíbrio influencia no efeito tóxico ${ }^{2}$ e na atividade farmacológica ${ }^{3}$ de sistemas ácido-base. Grande parte da fundamentação teórica da Química Orgânica moderna é baseada nos efeitos da estrutura molecular no equilíbrio ácido-base. ${ }^{4}$

Dados obtidos a partir de espectros de absorção UV-Vis em diferentes valores de $\mathrm{pH}$, em um processo conhecido por titulação espectrofotométrica, são frequentemente usados na determinação das constantes de ionização de ácidos e bases orgânicas.

Ghasemi e colaboradores ${ }^{5}$ apresentaram um algoritmo iterativo eficiente para a determinação das constantes de ácidos polipróticos a partir desses dados. Caminho semelhante foi usado na inversão de constantes de velocidade em modelos cinéticos por Bijlsma e colaboradores. ${ }^{6}$ Este procedimento lida diretamente com todo espectro de absorção e envolve, em uma das etapas, a minimização de uma função resíduo em relação às constantes de equilíbrio. O método mostrou-se superior aos programas SQUAD, ${ }^{7,8}$ SPECFIT 3.2,10 e DATAN 2.1:11 no entanto, Ghasemi cita a necessidade de novos testes para generalizar a eficiência do método.

Os algoritmos testados por Ghasemi ${ }^{5}$ apresentam modificações em relação ao método de otimização, na definição da função resíduo e na forma que os dados são projetados numa base menor e representativa. Nos programas SQUAD, STAR ${ }^{12}$ e SPECFIT 3.2, métodos não lineares como Simplex, ${ }^{13}$ Gauss Newton ${ }^{14}$ e o algoritmo de Levenberg-Marquardt ${ }^{15,16}$ foram utilizados para minimizar a função resíduo. Atualmente, esses pacotes têm sido usados na recuperação

*e-mail: nelson.lemes@unifal-mg.edu.br de constantes de dissociação de compostos com atividade farmacológica comprovada. ${ }^{17-19}$

Em 2007, um algoritmo geral, baseado em modelos de redes neurais recorrentes, ${ }^{20-22}$ com a finalidade de resolver problemas dessa natureza foi apresentado. ${ }^{23}$ Nesse trabalho algumas aplicações são discutidas, como a inversão das constantes de velocidade em problemas de cinética química ${ }^{24} \mathrm{e}$ a recuperação das constantes de força a partir de dados de espectroscopia vibracional. ${ }^{25} \mathrm{O}$ algoritmo mostrou-se versátil, robusto e preciso na resolução desses problemas e, em alguns casos, superou os resultados encontrados pelo método Simplex e o algoritmo de Levenberg-Marquardt. Trabalhos recentes mostram a eficiencia desse método na resolução de problemas inversos em diferentes áreas. ${ }^{26,27}$

No presente trabalho, utilizou-se esse modelo generalizado de rede neural recorrente na determinação das constantes de ionização de um composto orgânico natural, a partir de dados de espectros de absorção UV-Vis em diferentes valores de $\mathrm{pH}$. Este método não linear ainda não foi explorado na resolução de problemas inversos em equilíbrio químico.

O composto estudado é a benzofenona natural tetraprenilada, 7-epiclusianona, que pode ser isolada de plantas do gênero Rheedia, família Guttiferae. ${ }^{28}$ Esta molécula tem apresentado várias atividades biológicas, como propriedades anti-HIV, ${ }^{29}$ atividades contra tripomastigotas de Trypanosoma cruzi in vitro, ${ }^{30}$ efeitos vasodilatadores em anéis aórticos de ratos, ${ }^{31}$ propriedades antianafiláticas, ${ }^{32}$ leishmanicidas ${ }^{33} \mathrm{e}$ antimicrobianas. ${ }^{34} \mathrm{~A}$ clara compreensão do comportamento biológico e físico-químico passa pela avaliação de sua constante de ionização. ${ }^{3}$

A molécula apresenta baixa solubilidade em água, impossibilitando a obtenção da constante de acidez ${ }_{\mathrm{w}}^{\mathrm{w}} K_{\mathrm{a}}$ por métodos diretos. Quando uma substância é praticamente insolúvel, sua constante de dissociação em água pura pode ser obtida por extrapolação de determinações efetuadas em misturas binárias com solventes orgânicos. ${ }^{35,36}$ Neste trabalho determinaram-se, pela primeira vez, as constantes para 7-epiclusianona em misturas binárias etanol-água a partir de dados espectrofotométricos. 


\section{TEORIA}

\section{Método iterativo}

O método descrito por Ghasemi ${ }^{5}$ é baseado na minimização de uma função custo, definida por

$$
R=\frac{1}{2} \sum_{j, l} e_{j l}^{2}=\frac{1}{2} \sum_{j, l}\left(A_{j l}^{c a l}-A_{j l}\right)^{2},
$$

em relação às constantes de equilíbrio termodinâmico, com a restrição $\mathbf{A}=\mathbf{C P}^{T}$. Na Equação 1, $A_{j l}^{c a l}$ representa a absorbância calculada para o $j$-ésimo valor de $\mathrm{pH}$ e o $l$-ésimo comprimento de onda. As quantidades $A_{j l}$ são os respectivos valores experimentais.

As constantes de equilíbrio e as concentrações iniciais das espécies envolvidas determinam a direção em que a reação passa a ocorrer e a composição final da mistura no equilíbrio. ${ }^{37}$ Portanto, com o conhecimento de um valor aproximado das constantes de equilíbrio, a matriz $\mathbf{C}$ é determinada. A matriz $\mathbf{C}$ contém a concentração das $M$ espécies em equilíbrio, em $J$ valores diferentes de $\mathrm{pH}$.

Obtendo-se os espectros UV-Vis da solução, para uma faixa de $\mathrm{pH}$, tem-se a matriz absorbância A. A matriz A contém numa coluna a absorbância para $L$ comprimentos de onda e pH fixo. Uma linha de A apresenta a absorbância para vários valores de $\mathrm{pH}$ e comprimento de onda fixo.

Assumindo a lei de Beer-Lambert, ${ }^{1} \mathbf{A}=\mathbf{C P}^{T}$, a matriz contendo os espectros puros pode ser determinada por

$$
\mathbf{P}^{T}=\left(\mathbf{C}^{T} \mathbf{C}\right)^{-1} \mathbf{C A},
$$

em que $\left(\mathbf{C}^{T} \mathbf{C}\right)^{-1} \mathbf{C}^{T}$ representa a inversa generalizada da matriz $\mathbf{C}$ e $\mathbf{P}^{T}$ contém os espectros puros das espécies no equilíbrio. ${ }^{38}$ Neste caso, o espectro de absorbância total é decomposto nos espectros puros das espécies no equilíbrio.

A reconstrução do espectro de absorbância da mistura é obtida por

$$
\mathbf{A}^{c a l}=\mathbf{C P}^{T}
$$

Estas etapas são repetidas até se encontrar valores para as constantes de equilíbrio que minimizem a diferença entre o espectro experimental e calculado. Na busca sistemática das constantes ótimas podem ser utilizados diferentes métodos, como redes neurais recorrentes.

\section{Análise da componente principal}

A análise da componente principal permite colocar os dados experimentais numa base representativa, reduzindo a dimensão do problema e minimizando os efeitos dos erros experimentais. ${ }^{39}$

A decomposição em valor singular ${ }^{14}$ pode ser usada para encontrar uma base adequada. Transforma-se $\mathbf{A}$ no produto $\mathbf{U} \Sigma \mathbf{V}^{T}$, em que $\mathbf{U}$ e V são matrizes ortonormais e $\Sigma$ uma matriz diagonal. Os elementos da diagonal da matriz $\Sigma$ são chamados de valores singulares e as colunas das matrizes $\mathbf{U}$ e $\mathbf{V}$ são chamadas de vetores singulares. Os vetores singulares são representados por $\mathbf{u}_{\mathrm{i}} \mathrm{e} \mathbf{v}_{\mathrm{i}}$, e os valores singulares por $\sigma_{\mathrm{i}}$. O conjunto de valores singulares, em ordem decrescente, é chamado de espectro de valores singulares.

O espectro de valores singulares decai a zero. Para um sistema bem determinado e de posto completo existe uma queda acentuada para zero, em $i=k$, valor denominado posto da matriz. Para um sistema mal determinado não existe uma mudança característica no espectro de valores singulares e o posto da matriz corresponde ao número de componentes da base que contribuem significativamente para o valor de $\mathbf{A}$,

$$
\mathbf{A}=\sum_{i}^{k} \mathbf{t}_{i} \mathbf{v}_{i}^{T}+\mathbf{E}^{\prime}=\mathbf{A}^{(k)}+\mathbf{E}^{\prime}
$$

em que $\mathbf{T}=\mathbf{U} \Sigma$ e $\mathbf{E}^{\prime}$ representa o erro cometido ao desprezar os termos $k+1 \leq i \leq L$. As matrizes $\mathbf{T}$ e $\mathbf{V}^{T}$ não estão diretamente relacionadas com as matrizes $\mathbf{C}$ e $\mathbf{P}^{T}$. Introduzindo a matriz $\mathbf{Y}$, tal que $\mathbf{A}=\mathbf{C} \mathbf{P}^{T}$ $=\mathbf{C}\left(\mathbf{Y} \mathbf{Y}^{-1}\right) \mathbf{P}^{T}=(\mathbf{C Y})\left(\mathbf{Y}^{-1} \mathbf{P}^{T}\right)=\mathbf{T} \mathbf{V}^{T}$, tem-se $\mathbf{T}=\mathbf{C Y}$ e $\mathbf{V}^{T}=\mathbf{Y}^{-1} \mathbf{P}^{T}$.

Usando a análise da componente principal o algoritmo iterativo apresentado deve ser modificado. Inicialmente, com o conhecimento de um valor aproximado das constantes de equilíbrio, a matriz $\mathbf{C}$ pode ser calculada. A matriz $\mathbf{Y}$ é definida por $\mathbf{Y}=\left(\mathbf{C}^{T} \mathbf{C}\right)^{-1} \mathbf{C}^{T} \mathbf{T}$. Estas etapas são repetidas até se encontrar valores para as constantes de equilíbrio que minimizem o resíduo $R$, definido por

$$
R=\frac{1}{2} \sum_{j, l} e_{j l}^{2}=\frac{1}{2} \sum_{j, l}\left((C Y)_{j l}-T_{j l}\right)^{2} .
$$

Finalmente, os espectros puros são dados por $\mathbf{P}^{T}=\mathbf{Y} \mathbf{V}^{T}$.

Este procedimento aumenta a eficiência do algoritmo. A matriz T contém $k \times L$ elementos, um número menor que a matriz completa A, de dimensão $J \times L$.

\section{Redes neurais artificiais}

Neste trabalho utilizou-se um modelo generalizado de Hopfield, ${ }^{23}$ acoplado ao método iterativo ${ }^{5}$ na determinação das constantes de ionização de um ácido orgânico natural.

As redes neurais artificiais são técnicas computacionais inspiradas na estrutura neuronal. Neste trabalho foi implementada uma rede de $z$ neurônios em uma única camada, com uma função de ativação não linear $q$, em que o estado do neurônio $i$ no tempo $\tau$ é representado por uma variável contínua $u_{i}(\tau)$. No instante inicial, o estado do neurônio é definido por $u_{i}=q\left(K_{\mathrm{a} i}\right)$ para um valor aproximado da constante $K_{\mathrm{a} i}$. O estado dos neurônios é atualizado ao longo do tempo e a constante determinada pela expressão $K_{\mathrm{a} i}=q^{-1}\left(u_{\mathrm{i}}\right)$. A dinâmica da rede é descrita pelo sistema de equações diferenciais acopladas

$$
\frac{d u_{i}}{d \tau}=-\frac{\partial R}{\partial K_{\mathrm{a}_{i}}}=-\sum_{j l} e_{j l} \frac{\partial A_{j l}^{c a l}}{\partial K_{\mathrm{a}_{i}}}, \text { para } 1 \leq i \leq z .
$$

Neste modelo, o estado do neurônio $i$, no tempo $\tau+d \tau$, depende do estado de todos os neurônios no tempo $\tau$. A Equação 6 pode ser considerada uma generalização do modelo de Hopfield. ${ }^{20-22}$

A função de ativação $q$ pode ser apropriadamente escolhida para garantir que a rede sempre caminhe para um estado de menor resíduo, $d R / d \tau<0$. Derivando a função resíduo (Equação 1), em relação ao tempo, tem-se

$$
\frac{d R}{d \tau}=\sum_{i} \sum_{j, l} e_{j l} \frac{\partial A_{j l}^{c a l}}{\partial K_{\mathrm{a}_{i}}} \frac{d K_{\mathrm{a}_{i}}}{d u_{i}} \frac{d u_{i}}{d \tau}=-\sum_{i}\left\{\frac{d K_{\mathrm{a}_{i}}}{d u_{i}}\left(\frac{d u_{i}}{d \tau}\right)^{2}\right\} .
$$

Como $\left(d u_{i} / d \tau\right)^{2}>0$ e $d R / d \tau<0$, conclui-se que $d K_{\mathrm{a}_{i}} / d u_{i}>0$. Neste caso, uma escolha apropriada seria

$$
K_{\mathrm{a}_{i}}=q^{-1}\left(u_{i}\right)=\tanh \left(u_{i}\right),
$$

para a qual $d K_{\mathrm{a}_{i}} / d u_{i}=\sec ^{2}\left(u_{i}\right)>0$.

\section{PARTE EXPERIMENTAL}

A 7-epiclusianona foi obtida de Garcinia brasiliensis, conforme metodologia descrita por Derogis e colaboradores. ${ }^{40} \mathrm{~A}$ partir do epicarpo dos frutos, previamente secos e pulverizados, procedeu-se à 
extração em soxhlet com n-hexano por $24 \mathrm{~h}$. O extrato foi concentrado sob pressão reduzida para se obter o extrato hexânico do epicarpo. ${ }^{28,40}$ O extrato foi cromatografado em coluna de sílica gel (230-400 mesh) utilizando eluentes com misturas de polaridades crescentes de nhexano/acetato de etila e acetato de etila/etanol. As frações foram agrupadas conforme similitude na cromatografia de camada delgada. A 7-epiclusianona foi identificada pelo uso de padrões e por várias técnicas espectrométricas (IV, UV, EM e RMN).

A partir de soluções estoque de 7-epiclusianona 4,66 x 10-4 mol $\mathrm{L}^{-1}$ em etanol e $\mathrm{NaCl} 0,100 \mathrm{~mol} \mathrm{~L}^{-1}$ em água, preparou-se $50,0 \mathrm{~mL}$ das soluções a serem tituladas nas respectivas composições 40,0; 45,0; 50,0; 55,0 e 60,0\% etanol (m/v), com concentração final de 7-epiclusianona $4,66 \times 10^{-5} \mathrm{~mol} \mathrm{~L}^{-1}$ e $\mathrm{NaCl} 0,0100 \mathrm{~mol} \mathrm{~L}^{-1}$.

Utilizou-se um espectrofotômetro duplo feixe UV/Vis, Shimadzu, modelo UV-2550 equipado com controlador de temperatura, Shimadzu, TCC-240A, cubetas de quartzo de caminho óptico 1,0 $\mathrm{cm}$, potenciômetro Metrohm, $827 \mathrm{pH}$ lab, acoplado a um eletrodo de vidro com eletrodo de referência interna $\mathrm{Ag} / \mathrm{AgCl}$.

$\mathrm{O}$ pHmetro foi calibrado com tampões aquosos, para as leituras efetuadas nas misturas binárias, portanto obtém-se o $\mathrm{pH}$ da mistura (s) na escala aquosa (w), representado por ${ }_{\mathrm{w}}^{\mathrm{s}} \mathrm{pH}$. Consequentemente, os valores de $\mathrm{p} K_{\mathrm{a}}$ são obtidos na escala aquosa ${ }_{\mathrm{w}}^{\mathrm{s}} \mathrm{p} K_{\mathrm{a}} \cdot{ }^{41-43}$

Os espectros de absorção UV-Vis, em misturas binárias etanolágua, foram obtidos para valores de ${ }_{w}^{\mathrm{s}} \mathrm{pH}$ entre 3 e 12 à $25,0^{\circ} \mathrm{C}$. Para as variações no ${ }_{w}^{s} \mathrm{pH}$ utilizaram-se soluções de $\mathrm{HCl}$ e $\mathrm{NaOH}$, nas concentrações de 1,0 e $0,1 \mathrm{~mol} \mathrm{~L} \mathrm{~L}^{-1}$. $\mathrm{O}{ }_{\mathrm{w}}^{\mathrm{s}} \mathrm{pH}$ foi reduzido até não haver mudanças significativas no espectro de varredura, em seguida coletaram-se os espectros com o aumento do ${ }_{w}^{\mathrm{s}} \mathrm{pH}$, usando o mesmo critério.

A inversão da constante de equilíbrio é feita utilizando o método iterativo, Equações 2 e 3, na minimização por rede, Equação 6, do funcional $R$, Equação 5. A análise da componente principal, Equação 4 , é usada no cálculo de $R$. Os programas foram desenvolvidos pelos autores utilizando o ambiente de programação vetorial MatLab ${ }^{\circledR}$.

\section{RESULTADOS E DISCUSSÃO}

A Figura 1 apresenta a matriz A para solução de composição $40,0 \%$ etanol (m/v); abaixo desse percentual a 7-epiclusianona apresenta baixa solubilidade. Com o aumento do ${ }_{w}^{\mathrm{s}} \mathrm{pH}$ observa-se o aumento da absorbância em regiões abaixo de 299 nm, devido ao aumento da quantidade da espécie ionizada na mistura. A espécie ionizada apresenta maiores absortividades molares que a espécie não ionizada nessa região. Em regiões acima de 299 nm observa-se

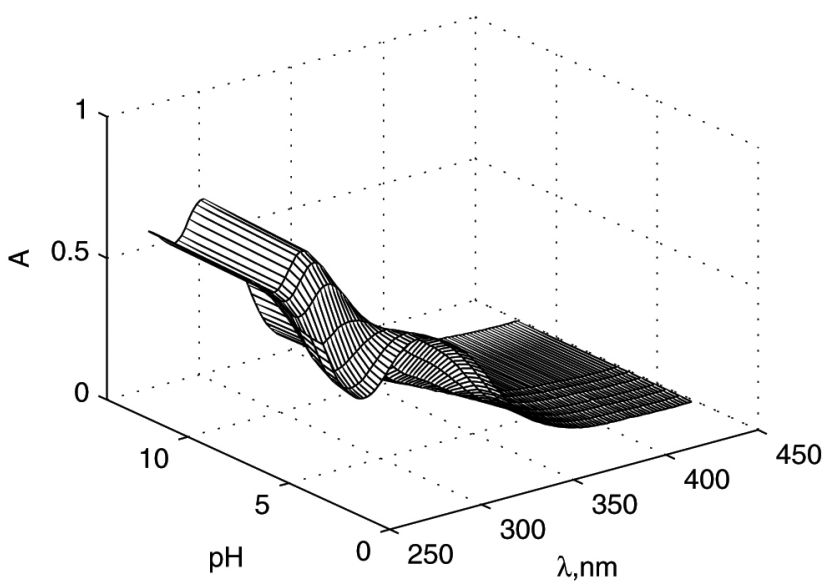

Figura 1. Espectros experimentais, 7-epiclusianona 4,66 $\times 10^{-5} \mathrm{~mol} \mathrm{~L}^{-1}, \mathrm{NaCl}$ $0,01 \mathrm{~mol} \mathrm{~L}^{-1}, 25,0{ }^{\circ} \mathrm{C}, 40,0 \% \mathrm{EtOH}(\mathrm{m} / \mathrm{v})$ o decréscimo da absorbância com o aumento do ${ }_{w}^{\mathrm{s}} \mathrm{pH}$, devido à diferença de absorvidade das duas espécies nesta região. Para todas as composições etanol-água observa-se um ponto isosbéstico próximo de $299 \mathrm{~nm}$ e aproximadamente os mesmos espectros puros.

O método iterativo utiliza como conhecimento a priori o número de hidrogênios ionizáveis, para que se possam determinar as equações que levam à matriz $\mathbf{C}$. No caso da molécula de 7-epiclusianona uma simples inspeção da sua estrutura (Figura 2) é suficiente para inferir a quantidade de hidrogênios ionizáveis, no entanto, para o caso geral pode-se obter esta variável a partir da decomposição em valor singular da matriz A.<smiles>CC(C)=CC[C@@H]1C[C@@]2(CC=C(C)C)C(=O)C(C(=O)c3ccccc3)=C(O)[C@@](CC=C(C)C)(C1=O)C2(C)C</smiles>

Figura 2. Estrutura química para 7-epiclusianona

Se $z$ é o número de hidrogênios ionizáveis, existem $z+1$ componentes no equilíbrio. Em situações em que não há desvio da lei de BeerLambert, a matriz A pode ser expressa como uma combinação linear dos espectros puros. Da mesma forma, os vetores colunas da matriz C podem representar uma base completa, $\mathbf{a}=p_{1} \mathbf{c}_{1}+p_{2} \mathbf{c}_{2}+p_{3} \mathbf{c}_{3}+\ldots$, com apenas $M$ vetores. Logo, o posto da matriz $\mathbf{A}$ menos um é igual ao número de constantes que há para determinar.

A primeira etapa do tratamento de dados envolve a escolha de uma base apropriada, usando a decomposição em valor singular da matriz absorbância. $\mathrm{O}$ espectro de valores singulares desta matriz é apresentado na Figura 3, para o teor de 40,0\% etanol (m/v). O espectro não mostra nenhum salto acentuado para o valor zero, deixando o problema mal determinado, no entanto, com uma queda mais significativa entre o segundo e terceiro valor singular, $\sigma_{2} / \sigma_{3}=94,65$.

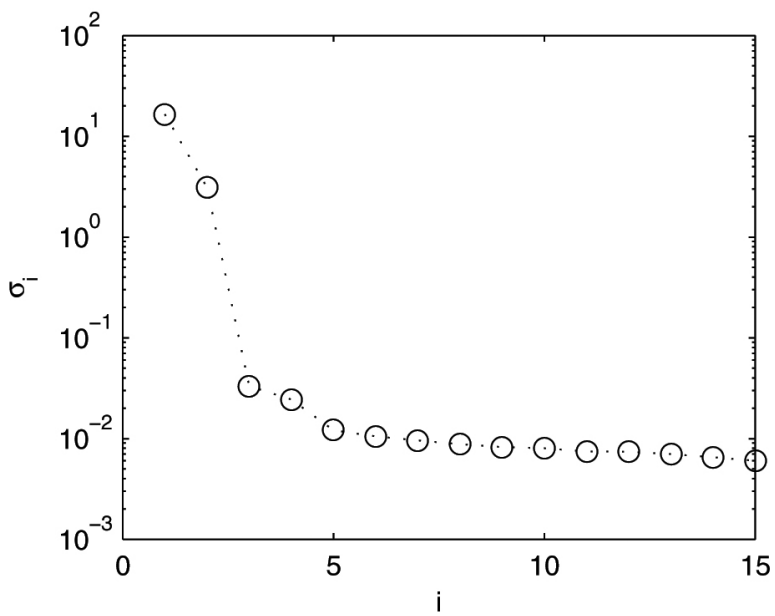

Figura 3. Espectro de valores singulares da matriz A, dimensão $201 \times 15$ 
A variância cumulativa para os dois primeiros componentes é de $99,25 \%$. A existência de 2 vetores significantes na base $v^{T}$ é suportado pelo ponto isosbéstico próximo de $299 \mathrm{~nm}$, característico de duas espécies que se interconvertem sem a formação de intermediários. ${ }^{44}$

O método de Runge-Kutta ${ }^{14}$ de quarta ordem com passo fixo foi usado na integração das equações acopladas (Equação 6), que descrevem a evolução dos estados dos neurônios na rede. Um valor inicial para o estado $u_{i}\left(\tau_{0}\right)$ do neurônio $i$ é definido pelo operador $q^{-1}\left(\mathrm{p} K_{\mathrm{a} i}\left(\tau_{0}\right)\right)$. $\mathrm{O} \mathrm{p} K_{\mathrm{a}_{i}}\left(\tau_{0}\right)$ representa uma estimativa inicial, neste caso avaliada em 7 , com resíduo $R$ igual a 2,9763. Outras estimativas iniciais convergiram para o mesmo resultado com $R^{*}$ inferior a $10^{-2}$. O passo de integração, $10^{-3}$, é escolhido para garantir a convergência e rapidez do método. A função de ativação foi definida de forma que a constante assumisse valores no intervalo de 0 a $14, \mathrm{p} K_{\mathrm{a}_{\mathrm{i}}}(\tau)=7\left\{1+\tanh \left[u_{i}(\tau)\right]\right\}$. Isto restringe o espaço de solução e aumenta a eficiência do algoritmo.

A derivada do resíduo em relação à constante, $\partial R / \partial \mathrm{p} K_{\mathrm{a} i}$, foi determinada numericamente via polinômio interpolador de grau 3. ${ }^{14} \mathrm{~A}$ função $R$ foi definida pela Equação 5. Neste caso, a rede foi acoplada ao método de análise da componente principal, através da função resíduo $R$ (Equação 5).

O tempo máximo de integração do sistema de equações diferenciais (Equação 6) foi determinado pelo princípio da discrepância. O método é baseado numa boa estimativa do erro envolvido na determinação experimental das absorbâncias, $R^{*}$. Ao longo da evolução dos estados dos neurônios o resíduo sempre decresce, conforme foi demonstrado. A solução ótima é encontrada quando o erro residual das absorbâncias no tempo $\tau^{*}$ é igual a $R^{*}, \mathrm{p} K_{\mathrm{a}_{i}}^{\text {otim }}=q^{-1}\left[u_{i}\left(\tau^{*}\right)\right]$.

A eficiência da rede, para determinar as constantes de equilíbrio a partir da titulação espectrofotométrica, foi comprovada pela comparação com o método Simplex. Neste caso, esperou-se a rede evoluir até reproduzir os resultados obtidos pelo método Simplex. Os valores de ${ }_{\mathrm{w}}^{\mathrm{s}} \mathrm{p} K_{\mathrm{a}}$ recuperados usando a rede como algoritmo de minimização foram iguais aos valores determinados pelo método Simplex até o sexto algarismo significativo. A Tabela 1 apresenta os valores de ${ }_{w}^{\mathrm{s}} \mathrm{p} K_{\mathrm{a}}$ obtidos.

Tabela 1. Constantes de acidez para a 7-epiclusianona em misturas etanolágua, à $25^{\circ} \mathrm{C}$

\begin{tabular}{cccc}
\hline$\% \operatorname{EtOH}(\mathrm{m} / \mathrm{v})$ & ${ }_{\mathrm{w}}^{\mathrm{s}} \mathrm{p} K_{\mathrm{a}}$ & $R^{*} / 10^{-3}$ & $\pm \delta$ \\
\hline 40,0 & 4,770 & 8,330 & 0,036 \\
45,0 & 4,892 & 9,352 & 0,035 \\
50,0 & 4,989 & 8,728 & 0,036 \\
55,0 & 5,124 & 6,810 & 0,034 \\
60,0 & 5,299 & 6,875 & 0,035 \\
\hline
\end{tabular}

Utilizando o princípio da discrepância pode-se avaliar os erros, $\delta_{i}$, nos valores dos ${ }_{\mathrm{w}}^{\mathrm{s}} \mathrm{p} K_{\mathrm{a}}$, de modo que qualquer solução $x$ no intervalo ${ }_{\mathrm{w}}^{\mathrm{s}} \mathrm{p} K_{\mathrm{a}} \pm \delta_{i}$ forneça um resíduo $R=\frac{1}{2} \sum_{j l}\left(A_{j l}^{\text {cal }}-A_{j l}^{\text {otim }}\right)^{2}$ menor ou igual $R^{*}$. A absorbância total calculada a partir dos valores ótimos encontrados das constantes é definida por $\mathbf{A}^{\text {otim }}$ e $\mathbf{A}^{\text {cal }}$ representa o valor calculado a apartir de $x$. O erro experimental na matriz absorbância permite o cálculo dos valores $\mathrm{de}_{\mathrm{w}}^{\mathrm{s}} \mathrm{p} K_{\mathrm{a}} \operatorname{com} 4$ algarismos significativos, portanto, são significativas as diferenças encontradas em função do teor de etanol (Tabela 1 ).

Os valores de ${ }_{\mathrm{w}}^{\mathrm{s}} \mathrm{p} K_{\mathrm{a}}$ nas misturas binárias mostram uma relação linear com a composição da mistura,

$$
{ }_{\mathrm{w}}^{\mathrm{s}} \mathrm{p} K_{\mathrm{a}}=m \times(\% \mathrm{~m} / \mathrm{v})+b,
$$

em que $b={ }_{\mathrm{w}}^{\mathrm{w}} \mathrm{p} K_{\mathrm{a}}$ indica o $\mathrm{p} K_{\mathrm{a}}$ em água pura, \%m/v representa a fração massa por volume do solvente orgânico e ${ }_{w}^{\mathrm{s}} \mathrm{p} K_{\mathrm{a}} \mathrm{o}$ valor de $\mathrm{p} K_{\mathrm{a}}$ na composição correspondente. Esta relação linear foi observada em outros sistemas ${ }^{45-47}$ Segundo Ghasemi, ${ }^{47}$ a influência da composição da mistura pode ser explicada pela facilidade de solvatação dos íons pelo meio. À medida que se tem mais água, há uma maior capacidade de solvatação estabilizando os íons formados na ionização, situação em que os produtos da ionização são mais favoráveis. Neste caso, ocorre um decréscimo do ${ }_{\mathrm{w}}^{\mathrm{s}} \mathrm{p} K_{\mathrm{a}}$. A reta que minimiza o erro quadrático dos dados da Tabela 1 é dada por ${ }_{\mathrm{w}}^{\mathrm{s}} \mathrm{p} K_{\mathrm{a}}=2,6156 \times 10^{-2} \times(\% \mathrm{~m} / \mathrm{v})$ $+3,7063\left(r^{2}=0.98768\right)$. Portanto, no limite de uma solução diluída em etanol, tem-se um ${ }_{\mathrm{w}}^{\mathrm{w}} \mathrm{p} K_{\mathrm{a}}=3,706$.

A determinação da constante de equilíbrio por métodos tradicionais utiliza dados experimentais em um único comprimento de onda. No método de inflexão ${ }^{48}$ é necessário um grande número de dados experimentais em $\mathrm{pH}$ próximo do $\mathrm{p} K_{\mathrm{a}}$. No caminho estabelecido pela Equação Henderson-Hasselbalch ${ }^{48}$ é necessário o conhecimento de informações dos espectros puros. Nesses métodos, a proximidade dos valores de $\mathrm{p} K_{\mathrm{a}}$ em sistemas complexos diminui a acurácia dos resultados. O caminho proposto neste trabalho utiliza dados experimentais em todo o espectro de absorção, o que reduz consideravelmente o efeito do ruído e leva a resultados finais mais precisos, conforme verificado na Figura 4.

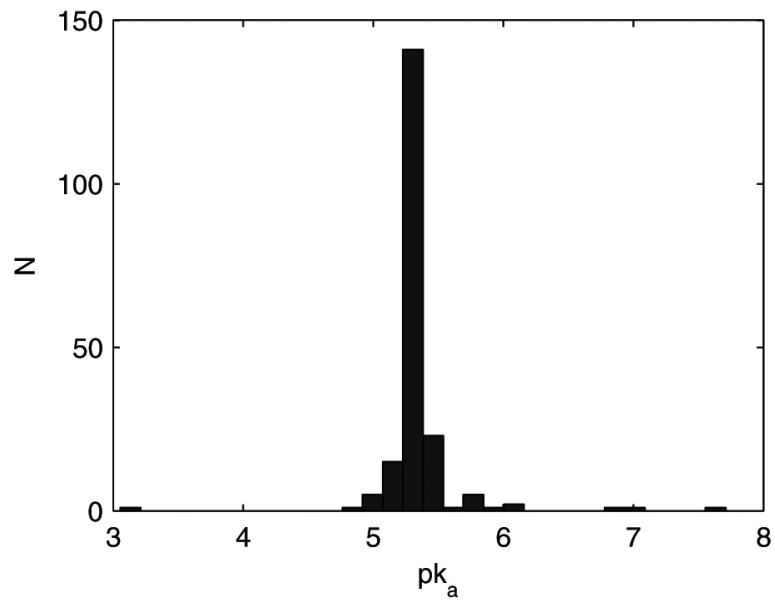

Figura 4. Histograma dos valores de ${ }_{w}^{s} p K_{a}$ obtidos pela inversão, a partir de dados experimentais em um único comprimento de onda. Foram obtidos 201 valores de ${ }_{w}^{s} p K_{a}$ com dados experimentais coletados na região entre 250 e $450 \mathrm{~nm}$

A Figura 4 apresenta o histograma dos valores de ${ }_{\mathrm{w}}^{\mathrm{s}} \mathrm{p} K_{\mathrm{a}}$ obtidos pela inversão, a partir de dados experimentais em um único comprimento de onda, usando as Equações 1-3, 6 e condições descritas anteriormente. Foram obtidos 201 valores de ${ }_{\mathrm{w}}^{\mathrm{s}} \mathrm{p} K_{\mathrm{a}} \mathrm{com}$ dados experimentais coletados na região entre 250 e $450 \mathrm{~nm}$, com uma média de 5,34 $\pm 0,32$. Este resultado mostra que a escolha apropriada do comprimento de onda é importante na recuperação da constante. Os critérios desta escolha podem ser apropriadamente definidos pela análise sensitiva da absorbância total em relação à constante de equilíbrio.

Os espectros de absortividades molares recuperados são concordantes com os resultados experimentais, uma vez que o produto dos vetores absortividade pela concentração analítica conduz aos espectros experimentais em meio bastante ácido e fortemente básico (Figura 5). Nesta condição tem-se praticamente os espectros puros para $\mathrm{HL}$ e $\mathrm{L}^{-}$. Ainda na Figura 5, verifica-se um ponto isosbéstico na mesma região de comprimento de onda $(299 \mathrm{~nm})$ para os dados experimentais e calculados. A composição do solvente tem um efeito pequeno em cada espectro puro, aproximadamente os espectros de 
absortividades molares são semelhantes aos recuperados para as demais composições etanol-água.

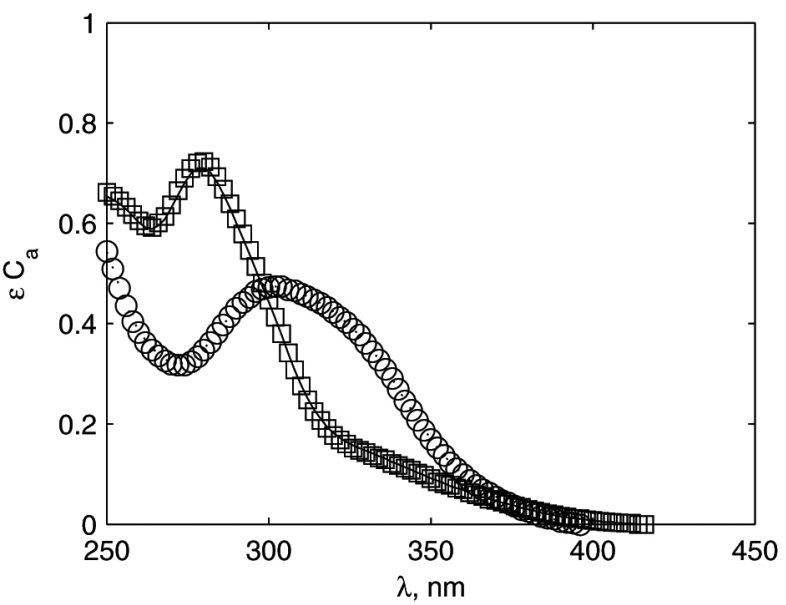

Figura 5. Absortividades molares obtidas: quadrados para espécie $H L e$ círculos para espécie $L^{-}$. As linhas contínua e pontilhada representam os dados experimentais em meio bastante ácido e fortemente básico, em que se têm praticamente os espectros puros para $H L$ e $L^{-}$, respectivamente

Para entender melhor os efeitos dos dados experimentais na inversão da constante de equilíbrio constrói-se a matriz sensibilidade. A matriz sensibilidade, definida por $S_{j l}=\frac{d A_{j l}}{d K_{\mathrm{a}}}$, é apresentada na Figura 6 através das linhas de contorno. A matriz sensibilidade foi calculada por diferenças finitas de $10^{-3}$ no ${ }_{\mathrm{w}}^{\mathrm{s}} \mathrm{p} K_{\mathrm{a}}$. Ela representa o efeito na absorbância total de uma mudança infinitesimal na constante de equilíbrio, em função do ${ }_{w}^{\mathrm{s}} \mathrm{pH}$ e comprimento de onda. ${ }^{49-51}$ Comparando com os espectros puros da Figura 5 observa-se que a maior sensibilidade ocorre na faixa de comprimentos de onda em que a diferença de absorvidade molar das espécies é maior. No comprimento de onda próximo de $299 \mathrm{~nm}$, a absorbância total não muda com o $\mathrm{pH}$ (ponto isobéstico), neste caso a sensibilidade é zero. Em relação ao $\mathrm{pH}$, a maior sensibilidade ocorre em valores próximos do ${ }_{\mathrm{w}}^{\mathrm{s}} \mathrm{p} K_{\mathrm{a}}$.

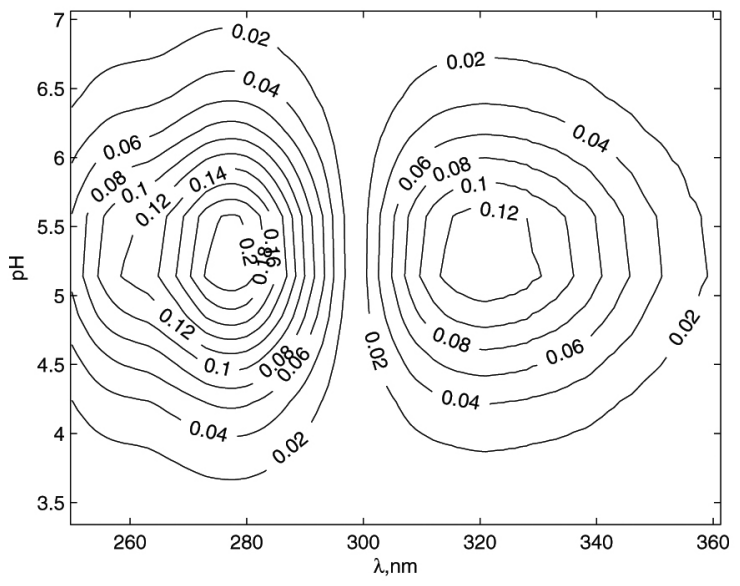

Figura 6. Efeito na absorbância total de uma mudança infinitesimal na constante de equilíbrio, em função do ${ }_{w}^{s} \mathrm{pH}$ e comprimento de onda

\section{CONCLUSÕES}

Uma rede neural dinâmica foi aplicada na determinação de constantes de equilíbrio a partir de espectros de absorção UV-Vis, em diferentes valores de ${ }_{\mathrm{w}}^{\mathrm{s}} \mathrm{pH}$.
O algoritmo utilizado é estável, rápido e de fácil implementação. Os resultados apresentam concordância com as constantes obtidas a partir de métodos testados na literatura. Os valores de ${ }_{\mathrm{w}}^{\mathrm{s}} \mathrm{p} K_{\mathrm{a}}$ recuperados usando a rede como algoritmo de minimização foram iguais aos determinados pelo método Simplex até o sexto algarismo significativo. O decréscimo do erro ao longo da evolução da rede, conforme demonstrado para a função de ativação $\mathrm{p} K_{\mathrm{a}_{i}}(\tau)=7\left\{1+\tanh \left[u_{i}(\tau)\right]\right\}$, garante a acurácia da solução encontrada.

Este modelo de rede neural não havia sido utilizado acoplado ao método de análise da componente principal. A representação dos dados experimentais numa base menor aumenta a eficiência da rede ao diminuir as dimensões das matrizes envolvidas no cálculo do resíduo. Outro procedimento que aumenta a precisão dos resultados é a pré-seleção dos dados com base na análise sensitiva.

O método apresentado pode ser facilmente aplicado à solução de problemas inversos em cinética química. Nesse problema os espectros são obtidos ao longo do tempo e a matriz $\mathbf{C}$ é construída a partir da solução de um conjunto de equações diferenciais acopladas.

Com o algoritmo descrito determinaram-se para a 7-epiclusianona, benzofenona natural com importantes atividades biológicas os espectros de absortividade molar das formas ácida e básica, as constantes de acidez em misturas etanol-água e, através da extrapolação dos valores obtidos, o valor do $\mathrm{p} K_{\mathrm{a}}=3,706$ para água pura.

\section{AGRADECIMENTOS}

À FAPEMIG e ao CNPq pelo suporte financeiro.

\section{REFERÊNCIAS}

1. Skoog, D. A.; West, D. M.; Holler, F. J.; Fundamentals of Analytical Chemistry, $8^{\text {th }}$ ed., Saunders College Publishing: Philadelphia, 1988.

2. Zhao, Y. H.; Yuan, L. H.; Wang, L. S.; Bull. Environ. Contam. Toxicol. 1996, 57,242

3. Rochester, H.; Acidity Functions; Academic Press: New York, 1971.

4. Sykes, P.; A guidebook to mechanism in organic chemistry; $6^{\text {th }}$ ed., John Wiley \& Sons: New York, 1985.

5. Ghasemi, J.; Nayebi, Sh.; Kubista, M.; Sjogreen, B.; Talanta 2006, 68, 1201.

6. Bijlsma, S.; Boelens, H. F. M.; Hoefsloot, H. C. J.; Smilde, A. K.; Anal. Chim. Acta 2000, 419, 197.

7. Leggett, D. J.; McBryde, W. A. E.; Anal. Chem. 1975, 47, 1065.

8. Leggett, D. J.; Anal. Chem. 1977, 49, 276.

9. Gammp, H.; Maeder, M.; Meyer, J. Ch.; Zuberbuhler, A. D.; Talanta 1985, 32, 95 .

10. Gammp, H.; Maeder, M.; Meyer, J. Ch.; Zuberbuhler, A. D.; Talanta 1985, 32, 257.

11. Kubista, M.; Sjöback, R.; Nygren, J.; Anal. Chim. Acta 1995, 302, 121.

12. Beltran, J. L.; Codony, R.; Prat, M. D.; Anal. Chim. Acta 1993, 276, 441.

13. Lagarias, J. C.; Reeds, J. A.; Wright, M. H.; Wright, P. E.; SIAM J. Optim. 1998, 9, 112.

14. Forsythe, G. E.; Malcolm, M. A.; Moler, C. B.; Computer Methods for Mathematical Computations, Prentice-Hall: New Jersey, 1977.

15. Levenberg, K.; Q. Appl. Math. 1994, 2, 164.

16. Marquardt, D.; SIAM J. Optim. 1963, 11, 431.

17. Meloun, M.; Derencikova, Z.; Vrana, A.; J. Chem. Eng. Data 2010, 55, 2695.

18. Sanli, N.; Sanli, S.; Alsancak, G.; J. Chem. Eng. Data 2010, 55, 2707.

19. Sanli, S.; Altun, Y.; Sanli, N.; Alsancak, G.; Beltran, J. L.; J. Chem. Eng. Data 2009, 54, 3021.

20. Hopfield, J. J.; Proc. Natl. Acad. Sci.U.S.A. 1982, 79, 2554.

21. Hopfield, J. J.; Proc. Natl. Acad. Sci.U.S.A. 1984, 81, 3088.

22. Hopfield, J. J.; Tank, D. W.; Biol. Cybern. 1985, 52, 141. 
23. Lemes, N. H. T.; Borges, E.; Braga, J. P.; J. Braz. Chem. Soc. 2007, 18, 1342.

24. Lemes, N. H. T.; Borges, E.; Braga, J. P.; Chemom. Intell. Lab. Syst. 2009, 96, 84 .

25. Borges, E.; Lemes, N. H. T.; Braga, J. P.; Chem. Phys. Lett. 2006, 423, 357.

26. Sebastião, R. C. O.; Braga, J. P.; Virtuoso, L. S.; Borges, E.; Quim. Nova 2011, 34, 213.

27. Sebastião, R. C. O.; Braga, J. P.; Virtuoso, L. S.; Vello, K. A. S. F.; Pacheco, C. N.; Silva, L. H. M.; Braz. J. Phys. 2010, 40, 423.

28. Santos, M. H.; Nagem, T. J.; Oliveira, T. T.; Braz-Filho, R.; Quim. Nova 1999, 22, 654

29. Piccinelli, A. L.; Cuesta-Rubio, O.; Chica, M. B.; Mahmood, N.; Pagano, B.; Pavone, M.; Barone, V.; Rastrelli, L.; Tetrahedron 2005, 61, 8206.

30. Alves, R. O.; Romanha, A. J.; Santos, M. H.; Nagem, T. J.; Zani, C. L.; J. Nat. Prod. 1999, 62, 369.

31. Cruz, A. J.; Lemos, V. S.; Santos, M. H.; Nagem, T. J.; Cortes, S. F.; Phytomedicine 2006, 13, 442.

32. Neves, J. S.; Coelho, L. P.; Cordeiro, R. S. B.; Veloso, M. P.; Silva, P. M. R.; Santos, M. H.; Martins, M. A.; Planta Med. 2007, 73, 644.

33. Pereira, I. O.; Marques, M. J.; Pavan, A. L.R.; Codonho, B. S.; Barbiéri, C. L.; Beijo, L. A.; Doriguetto, A. C.; D’Martin, E. C.; Santos, M. H.; Phytomedicine 2010, 17, 339 .

34. Naldoni, F. J.; Claudino, A. L. R.; Cruz, J. W.; Chavasco, J. K.; Faria e Silva, P. M.; Veloso, M. P.; Santos, M. H.; J. Med. Food 2009, 12, 403.

35. Fallavena, P. R. B.; Schapoval, E. E. S.; Int. J. Pharm. 1997, 158, 109.
36. Kubista, M.; Sjöback, R.; Albinsson, B.; Anal. Chem. 1993, 65, 994.

37. Klotz, I. M.; Rosenberg, R. M.; Chemical Thermodynamics: Basic Concepts and Methods, John Wiley \& Sons: New Jersey, 2008.

38. Golub, G. H.; Vanloan, F.; Matrix Computations, John Hopkins University Press: Baitimore, 1983.

39. Maeder, M.; Neuhold, Y.-M. Em Data Handling in Science and Technology; Rutan, S.; Walczak, B., eds.; Elsevier: Amsterdam, 2007, vol. 26.

40. Derogis, P. B. M. C.; Martins, F. T.; Souza, T. C.; Moreira, M. E.; Souza Filho, J. D.; Doriguetto, A. C.; Souza, K. R. D.; Veloso, M. P.; Santos, M. H.; Magn. Reson. Chem. 2008, 46, 278.

41. Espinosa, S.; Bosch, E.; Rosés, M.; Anal. Chem. 2002, 74, 3809.

42. Bates, R. G.; Paabo, M.; Robinson, R. A.; J. Phys. Chem. 1963, 67, 1833.

43. Bates, R. G.; Determination of $p H$, Theory and Practice, $2^{\text {nd }}$ ed., Wiley Interscience: New York, 1972.

44. Elbergali, A.; Nygren, J.; Kubista, M.; Anal. Chim. Acta 1999, 379, 143.

45. Castro, G. T.; Giordano, O. S.; Blanco, S. E.; THEOCHEM 2003, 626 , 167.

46. Castro, G. T.; Ferretti, F. H.; Blanco, S. E.; Spectrochim. Acta, Part A 2005, 62, 657.

47. Ghasemi, J.; Lotfi, S.; Safaeian, M.; Niazi, A.; Ardakani, M. M.; Noroozi, M.; J. Chem. Eng. Data 2006, 51, 1530.

48. Harvey, D.; Modern Analytical Chemistry, McGraw-Hill: New York, 2000.

49. Rabitz, H.; Science 1989, 246, 221.

50. Ho, T.-S.; Rabitz, H.; J. Chem. Phys. 1989, 90, 1519.

51. Ho, T.-S.; Rabitz, H.; J. Chem. Phys. 1989, 91, 7590. 